\title{
Frecuencia de mutaciones en el gen de la usherina (USH2A) en 26 individuos colombianos con síndrome de Usher, tipo II
}

\author{
Greizy López¹, Nancy Yaneth Gelvez¹, Martalucía Tamayo ${ }^{1,2}$ \\ 1 Instituto de Genética Humana, Pontificia Universidad Javeriana, Bogotá, D.C., Colombia \\ 2 Fundación Oftalmológica Nacional, Bogotá, D.C., Colombia \\ Institución responsable: Instituto de Genética Humana, Pontificia Universidad Javeriana, Bogotá, D.C., \\ Colombia
}

Introducción. El síndrome de Usher se caracteriza por hipoacusia neurosensorial congénita, retinitis pigmentaria y disfunción vestibular. Es la causa más frecuente de sordo-ceguera en el mundo. Se divide en tres tipos clínicos y doce subtipos genéticos. El tipo II es la forma más común y cerca de $80 \%$ de los casos corresponden al subtipo 2 del síndrome de Usher.

Objetivo. Establecer la frecuencia de mutaciones en la isoforma corta del gen USH2A en individuos colombianos con síndrome de Usher, tipo II.

Materiales y métodos. Se estudiaron 26 individuos colombianos con diagnóstico clínico de síndrome de Usher, tipo II. Se hizo análisis de SSCP para los 20 exones que codifican para la isoforma corta y se secuenciaron los patrones anormales. Además, se secuenció el exón 13 en todos los individuos, ya que allí se encuentra la mutación más frecuente de este gen.

Resultados. La mutación más frecuente es la c.2299delG, correspondiente al 27 \% de la población. La segunda mutación identificada es la p.R334W, con una frecuencia de $15 \%$. Se identificó un nuevo cambio, el g.129G>T,en la región 5'UTR del gen, correspondiente al 4 \% de la población. Se identificaron cuatro cambios polimórficos, uno de ellos es una deleción nueva identificada en el exón 20.

Conclusiones. Se logró establecer que, al menos, 38 \% de la población analizada con síndrome de Usher, tipo II, presenta alguna mutación en la isoforma corta del gen de la usherina. El diagnóstico molecular se logró establecer en el $23 \%$.

Palabras clave: síndromes de Usher/genética, retinitis pigmentaria, pérdida auditiva neurosensorial; análisis de mutación de ADN, Colombia.

Mutational frequencies in usherin (USH2A gene) in 26 Colombian individuals with Usher syndrome type II

Introduction. Usher syndrome is a disorder characterized by progressive retinitis pigmentosa, prelingual sensory hearing loss and vestibular dysfunction. It is the most frequent cause of deaf-blindness in humans. Three clinical types and twelve genetic subtypes have been characterized. Type II is the most common, and among these cases, nearly $80 \%$ have mutations in the USH2A gene.

Objective. The aim of the study was to establish the mutational frequencies for the short isoform of USH2A gene in Usher syndrome type II.

Materials and methods. Twenty-six Colombian individuals with Usher syndrome type II were included. SSCP analysis for 20 exons of the short isoform was performed and abnormal patterns were sequenced. Sequencing of exon 13 of the USH2A gene was performed for all the individuals because the most frequent mutation is located in this exon.

Results. The most frequent mutation was c. $2299 \mathrm{delG}$, identified in the $27 \%(n=8)$ of the sample. The second mutation, p.R334W, showed a frequency of $15 \%$. A new variant identified in the 5'UTR region, g.129G > T, was present in 1 individual (4\%). Four polymorphisms were identified; one of them is a new deletion in exon 20 , first reported in this study.

Conclusions. Mutations in the usherin short isoform were identified in $38 \%$ of a sample of 26 USH2 cases. Molecular diagnosis was established in 7 of the 26.

Key words: Usher syndromes/genetics, retinitis pigmentosa; hearing loss, sensorineural; Genetics, DNA mutational analysis, Colombia. 
El síndrome de Usher es una enfermedad genética de herencia autosómica recesiva, que cursa con hipoacusia neurosensorial, retinitis pigmentaria progresiva y, en algunos casos, disfunción vestibular. Este síndrome es la causa más frecuente de sordoceguera en el mundo, y corresponde a $6 \%$ de la población congénitamente sorda y a $18 \%$ de toda la población con retinitis pigmentaria (1).

La prevalencia del síndrome de Usher se ha calculado en un rango de 3,5 a 6,2 casos por cada 100.000 habitantes (2); su frecuencia en Estados Unidos es de alrededor de 5 por 100.000 habitantes (1), en Escandinavia, de 3,0 por 100.000 (3) y en Colombia, de 3,2 por 100.000 habitantes, y corresponde a $9,6 \%$ de la población sorda y a 10 $\%$ de la población ciega (4-6).

Clínicamente se divide en tres tipos: el tipo I se caracteriza por sordera severa (sic.) congénita, ausencia de respuesta vestibular y aparición de retinitis pigmentaria progresiva en la primera década de la vida o a principios de la segunda; el tipo II, por sordera congénita de moderada a grave, respuesta vestibular normal, con inicio de retinitis pigmentaria progresiva en la segunda década de la vida, y el tipo III, por pérdida auditiva progresiva, respuesta vestibular variable y aparición de retinitis pigmentaria en edad variable (7).

El síndrome de Usher presenta una significativa heterogeneidad genética: a cada uno de los tres tipos clínicos del síndrome le corresponden subtipos genéticos.

Hasta el momento, se han identificado 12 loci asociados: siete responsables del síndrome de Usher de fenotipo 1 (USH1B-H), tres del síndrome de Usher de fenotipo 2 (USH2A, 2C y 2D) y dos del síndrome de Usher de fenotipo 3 (USH3A y 3B). Los tres genes responsables del fenotipo 2 ya han sido mapeados. De éstos, el primer gen identificado fue el USH2A, el cual codifica para la proteína usherina (8). Esta proteína es necesaria para el correcto mantenimiento de las células fotorreceptoras de la retina y para el desarrollo de las células ciliadas de la cóclea (9).

\footnotetext{
Correspondencia:

Martalucía Tamayo, Instituto de Genética Humana, Pontificia Universidad Javeriana, Carrera 7 № 40-62, edificio 32, Bogotá, D.C., Colombia

Teléfono: (571) 320 8320, extensión: 2788 o 2787; fax: (571) 320 8320, extensión: 2793

mtamayo@javeriana.edu.co

Recibido: 23/06/10; aceptado:30/09/10
}

Entre los tipos clínicos identificados hasta el momento, el de tipo II es la forma más común de síndrome de Usher, ya que se presenta en casi la mitad de los casos reportados (10-12). Algunos estudios han demostrado que de $74 \%$ a $83 \%$ de los casos con fenotipo 2, corresponden al subtipo $2 A$ (13-16). Inicialmente, se describió que el gen USH2A constaba de 21 exones (14), pero posteriormente se definió que existe una segunda isoforma que comprende 51 exones adicionales a los ya conocidos, para un total de 72 (17).

El objetivo de este trabajo fue identificar las mutaciones en el gen que codifica para la isoforma corta de la usherina (gen USH2A) en individuos colombianos con síndrome de Usher, tipo II, con el fin de establecer la frecuencia de las mutaciones propias de la población colombiana.

\section{Materiales y métodos}

\section{Población de estudio}

Se estudiaron 26 individuos colombianos con diagnóstico clínico de síndrome de Usher, tipo II, que fueron seleccionados en tamizaciones realizadas por el Instituto de Genética Humana de la Pontificia Universidad Javeriana y la Fundación Oftalmológica Nacional en institutos para ciegos y sordos de 11 ciudades del país.

A todos los individuos se les hizo examen de fondo de ojo, electrorretinograma, campimetría, angiografía, fotos de fondo de ojo y audiometría. Se tomaron muestras de $10 \mathrm{ml}$ de sangre periférica por punción venosa, previa firma del consentimiento informado.

De la población estudiada, cinco individuos pertenecían a familias "informativas" (familias, al menos, con dos individuos afectados $y$, al menos, un familiar sano en primer grado), en las cuales se había establecido previamente el fenotipo 2A por medio de análisis de haplotipos, y 21 individuos pertenecían a familias no "informativas" (familias con un número insuficiente de individuos sanos y afectados) de USH2.

\section{Pruebas moleculares}

El ADN se extrajo por medio de la técnica de fenolcloroformo. Los iniciadores que se utilizaron para la amplificación de los 21 exones del gen USH2A, se muestran en el cuadro 1.

\section{Análisis de secuenciación}

Se hizo el análisis de secuenciación bidireccional del exón 13 del gen USH2A para los 26 individuos del estudio. 
Cuadro 1. Iniciadores utilizados para la amplificación de los 21 exones de la isoforma corta del gen USH2A.

\begin{tabular}{|c|c|c|}
\hline Exó & Iniciadores & $\begin{array}{c}\text { Tamaño } \\
\text { del } \\
\text { fragmento } \\
(p b)\end{array}$ \\
\hline 1 & $\begin{array}{l}\text { F: 5' AAAGCTTGGGCAGCTCAGTTCCAA 3' } \\
\text { R: 5' ACACATGGATCCCTAATACTGTGC 3' }\end{array}$ & 307 \\
\hline 2 & $\begin{array}{l}\text { F: 5' AAAGGTGCAGCAGCTTTCCCTTGA 3' } \\
\text { R: 5' TGGGGACCTATGAAAGCTTATACC 3' }\end{array}$ & 775 \\
\hline 3 & $\begin{array}{l}\text { F: 5' GGTTGGATGCTGACCTTTTCTCTC 3' } \\
\text { R: 5' CTGCAGATTTTGTGAGTAGATGCC 3' }\end{array}$ & 304 \\
\hline 4 & $\begin{array}{l}\text { F: 5' CAGTCTTCCCAGCTGAACAAAGTA 3' } \\
\text { R: 5' GCCCTAGAAGATGAATACACGTAG 3' }\end{array}$ & 366 \\
\hline 5 & $\begin{array}{l}\text { F: 5' GTTCGATCAGTAAATAGGCTGGCC 3' } \\
\text { R: 5' GGAGTTCCTCAAGAGTAGCACTAG 3' }\end{array}$ & 514 \\
\hline 6 & $\begin{array}{l}\text { F: 5' AGCTCTTTGTCCTTACAGTCTCCC 3' } \\
\text { R: 5' GGGCATTTGTTGCAATAACCACAG 3' }\end{array}$ & 391 \\
\hline 7 & $\begin{array}{l}\text { F: 5' GGGCTATGAAGTTTCTGGATGTCT 3' } \\
\text { R: 5' ACCAGCCTAGAGAGCTAGCATACT 3' }\end{array}$ & 420 \\
\hline 8 & $\begin{array}{l}\text { F: 5' GTGTGGCTTTCTATGATAATCCTG 3' } \\
\text { R: 5' GCTTGAAATCTGGCTTGCTCTGAC 3' }\end{array}$ & 493 \\
\hline 9 & $\begin{array}{l}\text { F: 5' GAGTTGGTGTTCCTTAGGAGTGTG 3' } \\
\text { R: 5' GAACCTTAGGGTTTGGTTTCAG 3' }\end{array}$ & 376 \\
\hline 10 & $\begin{array}{l}\text { F: 5' GTGCTTTACTTCTGGTGAAAGGCC 3' } \\
\text { R: 5' TAGATAGAAGCACACAGGCCTCCA 3' }\end{array}$ & 344 \\
\hline 11 & $\begin{array}{l}\text { F: 5' GCTCTGGATCACTAAATTGGCAGG 3' } \\
\text { R: 5' GAGGATTTCCTGGCAAATGCAGTC 3' }\end{array}$ & 400 \\
\hline 12 & $\begin{array}{l}\text { F: 5' CTCCCTCCCTGTCTTGTACCTAAT 3' } \\
\text { R: 5' CCAGCCTGTCTTGAGCAAAGAAAT 3' }\end{array}$ & 394 \\
\hline 13 & $\begin{array}{l}\text { F: 5'CGTATCATCTGCAGTAGCATTG 3' } \\
\text { R: 5'AAGCCACAAACCAGAAACAGGG 3' }\end{array}$ & 822 \\
\hline 14 & $\begin{array}{l}\text { F: 5' GAGGCACCCAAGTCCAATGTAGAT 3' } \\
\text { R: 5' AGCCACCAAGCCGGGCAAAAAAAA 3' }\end{array}$ & 504 \\
\hline 15 & $\begin{array}{l}\text { F: 5' CAAGCCGTCTTACTCTACAATGCT 3' } \\
\text { R: 5' TCCCCTGTATGATGCTGCTTCACA 3' }\end{array}$ & 318 \\
\hline 16 & $\begin{array}{l}\text { F: 5' GAATCAGAAAGACCTCTTTGCTGG 3' } \\
\text { R: 5' ACACTCTGTGCCAGACAGAGGAAA 3' }\end{array}$ & 370 \\
\hline 17 & $\begin{array}{l}\text { F: 5' GCAATGATTCTTCACCAACTTGTG 3' } \\
\text { R: 5' TGACCAAAAAGGGGAATCTCAGCC 3' }\end{array}$ & 593 \\
\hline 18 & $\begin{array}{l}\mathrm{F}: \text { 5' TCCCTACCCTACCCCTAAAGTAAC 3' } \\
\text { R: 5' CCCCTAGAACTTGCTGCAAGTACT 3' }\end{array}$ & 539 \\
\hline 19 & $\begin{array}{l}\text { F: 5' GGGACGACATTCATTGTTTTGATG 3' } \\
\text { R: 5' GCCCTAAGCCAATTCTGAAAGGAC 3' }\end{array}$ & 264 \\
\hline 20 & $\begin{array}{l}\text { F: 5' CCCTCTGTCTGAATTCATGGAACT 3' } \\
\text { R: 5' AGCAATCAGAGTTAGTGAGGGAGG 3' }\end{array}$ & 371 \\
\hline 21 & $\begin{array}{l}\text { F: 5' CAGTTCCTAGAGCCATACAGATAC 3' } \\
\text { R: 5' AACATTTTGGAGCTTCGTGTCTCC 3' }\end{array}$ & 433 \\
\hline
\end{tabular}

El procedimiento utilizado para la secuenciación fue el siguiente: una vez obtenido el producto de PCR (Polymerase Chain Reaction), se purificó y se realizó la reacción de secuenciación con $2 \mu \mathrm{l}$ de mezcla de reacción, $2 \mu \mathrm{l}$ de solución tampón $5 \mathrm{X}, 1 \mu \mathrm{l}$ de iniciador y $5 \mu \mathrm{l}$ de producto purificado.
Las condiciones de temperatura fueron: $95^{\circ} \mathrm{C}$ por 10 segundos, $50{ }^{\circ} \mathrm{C}$ por 5 segundos y $60^{\circ} \mathrm{C}$ por 4 minutos, durante 35 ciclos. Se realizó la precipitación de la reacción de secuenciación por el método de etanol. Las análisis de secuenciación se realizaron en el ABI-PRISM 3100-Avant ${ }^{\mathrm{TM}}$ con el estuche de secuenciación BigDye terminator ${ }^{\mathrm{TM}}$, versión 3.1. Finalmente, se analizaron las secuencias con el software DNAstar ${ }^{\mathrm{TM}}$.

\section{Análisis de Single Strand Conformation Polymorphism}

Se hizo análisis de Single Strand Conformation Polymorphism (SSCP) para los 21 exones que codifican para la isoforma corta del gen USH2A. Las amplificaciones se llevaron a cabo en el termociclador icycler BIO-RAD ${ }^{\mathrm{TM}}$. Se utilizó el siguiente protocolo estándar: un ciclo inicial de $95^{\circ} \mathrm{C}$ por 5 minutos, 30 ciclos de $95^{\circ} \mathrm{C}$ por 1 minuto, la temperatura de anillamiento correspondiente en cada caso por 1 minuto y $72{ }^{\circ} \mathrm{C}$ por 1 minuto, seguidos por un ciclo de elongación final a $72{ }^{\circ} \mathrm{C}$ por 7 minutos. Una vez obtenidos los productos, se hizo análisis de SSCP para cada uno de los exones, en las cámaras DeCode y MiniProtean I/ de BIO$R A D$, según las condiciones de estandarización en cada caso.

Las condiciones generales para la preparación de la muestra fueron: a una alícuota de $8 \mu \mathrm{l}$ del producto, se agregaron $8 \mu \mathrm{l}$ de solución tampón de formamida. La mezcla fue desnaturalizada a $95^{\circ} \mathrm{C}$ por 5 minutos, colocada en hielo y sembrada en un gel de poliacrilamida no desnaturalizador, con glicerol al $10 \%$ y con diferentes concentraciones de poliacrilamida, que variaban entre $6 \%$ y $12 \%$, según las condiciones de cada exón. Las bandas se visualizaron con tinción de nitrato de plata. Los patrones anormales fueron secuenciados según el protocolo mencionado anteriormente.

\section{Base de datos}

La secuencia consenso utilizada para el gen USH2A se obtuvo de:

http://www.ensembl.org/Homo_sapiens/Transcript/ Summary?db=core;g=ENSG00000042781;r=1:21 5796236-216596738;t=ENST00000366942.

\section{Resultados}

Los resultados del análisis de mutaciones se presentan en el cuadro 2. Se identificó la mutación c.2299 delG (figura 1) en siete de los individuos analizados (27\%). En uno de ellos, en estado homocigoto, y en los seis restantes, en estado heterocigoto. 
Cuadro 2. Frecuencias de los cambios identificados en el gen USH2A.

\begin{tabular}{|c|c|c|c|}
\hline Exón & Cambio & $\begin{array}{l}\text { Frecuencia alélica en } \\
\text { propósitos (\%) }\end{array}$ & $\begin{array}{l}\text { Reporte en otras } \\
\text { poblaciones }\end{array}$ \\
\hline \multicolumn{4}{|c|}{ Mutaciones identificadas } \\
\hline 1 & g.129G $>\mathrm{T}$ & $(2 / 52)$ & No reportado \\
\hline 6 & c. $1000 \mathrm{C}>\mathrm{T}$, p.R334W & $(6 / 52)$ & $0-3(18)$ \\
\hline 13 & c.2299delG, p.E767FS & $(8 / 52)$ & $22-24(8)$ \\
\hline \multicolumn{4}{|c|}{$\begin{array}{l}\text { Polimorfismos o cambios } \\
\text { no patogénicos }\end{array}$} \\
\hline 3 & c.504A>G, p.T168T & $32,7 \quad(17 / 52)$ & rs4253963 (19) \\
\hline 11 & c.1931A>T, p.D644V & $7,7 \quad(4 / 52)$ & rs1805048 (19) \\
\hline 20 & c.4252-24_-13delCTTT & $1,9 \quad(1 / 52)$ & No reportado \\
\hline 21 & c. $4457 \mathrm{G}>\overline{\mathrm{A}}, \mathrm{p} . \mathrm{R} 1486 \mathrm{~K}$ & $75 \quad(39 / 52)$ & rs1805049 (19) \\
\hline
\end{tabular}
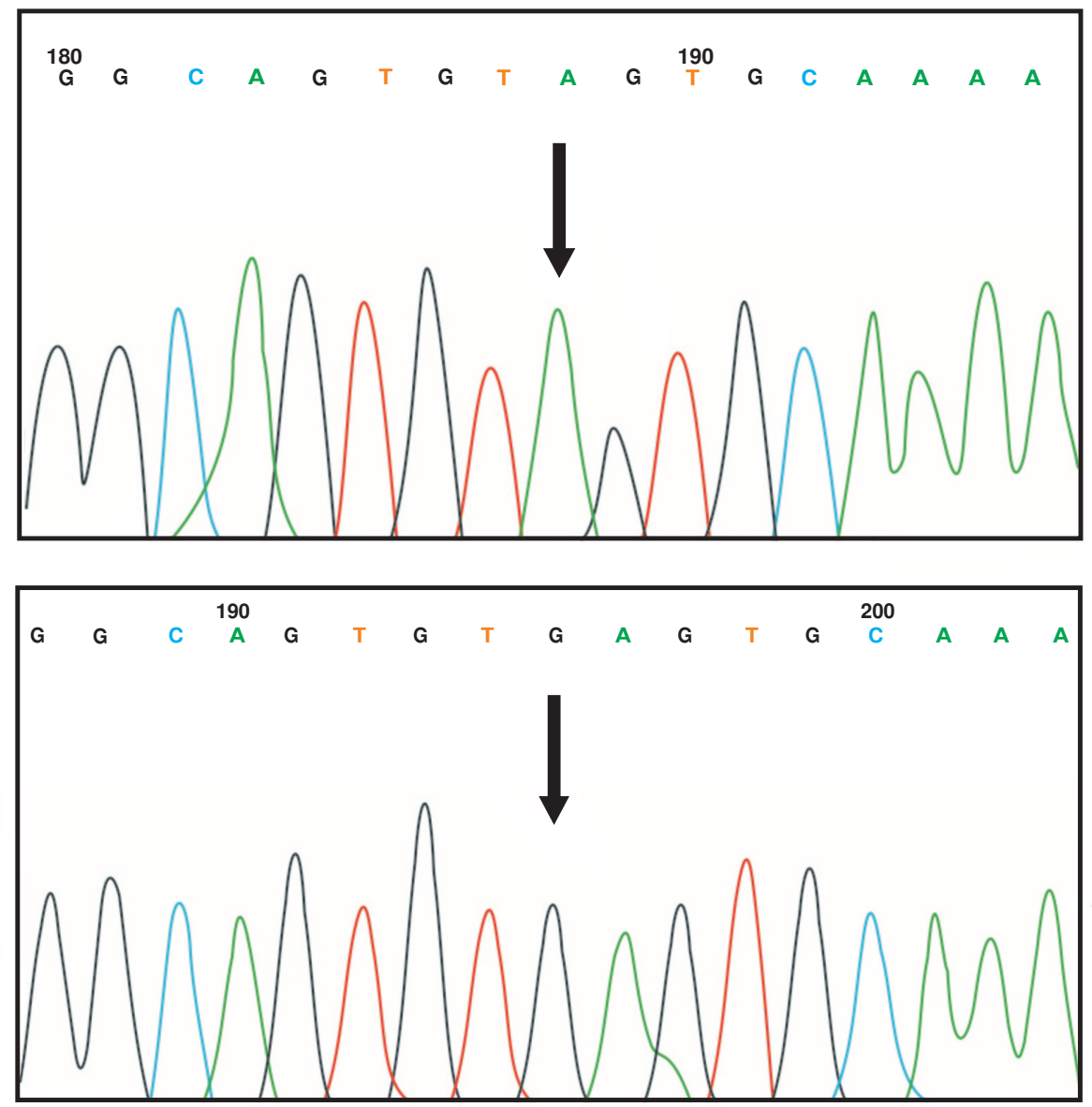

Figura 1. Electroferograma de la secuencia del exón 13 del gen USH2A. Arriba: la mutación c.2299delG en estado homocigoto. Abajo: secuencia sin la mutación.

El análisis de SSCP reveló patrones anormales en los exones 1, 3, 6, 11, 20 y 21 del gen USH2A. Por medio del análisis de secuenciación de los patrones anormales, se identificaron varios cambios.

En el exón 6 se identificó una mutación previamente reportada (17). Se trata de una transición $\mathrm{C} \rightarrow T$ en el nucleótido 1.000, que conduce al cambio de arginina por triptófano en el aminoácido
334, p.R334W. La mutación -que se identificó en $15 \%$ de la población- se encontró en dos individuos en estado homocigoto y en otros dos, en estado heterocigoto compuesto con la mutación c.2299delG.

En el exón 1 se identificó un cambio aún no reportado (figura 2) en una familia con fenotipo $2 \mathrm{~A}$. Se trata de una transversión $\mathrm{G}>\mathrm{T}$ en la posición 129 en la 
Figura 2. Análisis del cambio g.129G>T. A) Árbol genealógico de la familia. Los individuos afectados (en color negro) fueron homocigotos para el cambio, los individuos sanos (sin rellenar) fueron heterocigotos. B) Secuencia consenso del gen USH2A. Se observan los dos primeros exones del gen, de los cuales sólo el 2 es codificante. Se encuentra sombreada la región 5'UTR del gen. El cambio g.129G>T se encuentra resaltado en un cuadro.

B

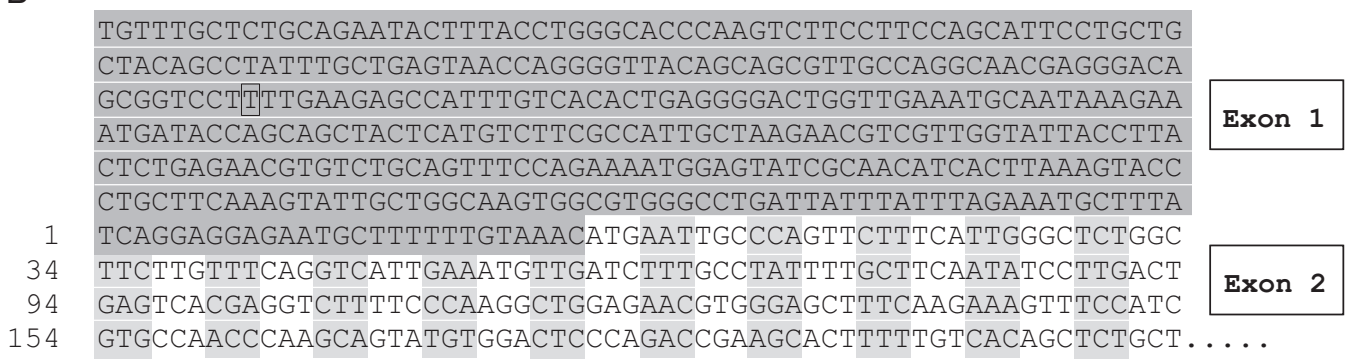

región 5'UTR del gen, a 259 pb corriente arriba del codón de inicio ATG. El cambio se encontró en estado homocigoto en los tres individuos afectados $\mathrm{y}$, en estado heterocigoto, en todos los demás miembros de la familia. El cambio no fue identificado en 100 controles sanos colombianos.

Se identificaron cuatro cambios polimórficos. El primero de ellos, c.504A>G, p.Thr168Thr en el exón 3 , se observó en 13 individuos en estado heterocigoto y en dos en estado homocigoto. Un segundo polimorfismo en el exón 11, c.1931A>T, p.D644V, estuvo presente en cuatro individuos en estado heterocigoto. En el exón 21 se identificó el cambio c.4457G>A, p.R1486K, en estado heterocigoto, en 9 individuos del estudio, y en estado homocigoto, en 15 individuos. El análisis de SSCP del exón 20 del gen USH2A reveló un cambio adicional en un individuo con fenotipo 2. Se trata de una deleción de tres repeticiones en tándem CTTT en la región intrónica flanqueante del extremo 5' (figura 3), que se encuentra en estado heterocigoto en el individuo afectado. Este cambio no se identificó en 100 controles sanos colombianos. Las frecuencias alélicas de estos cambios se pueden observar en el cuadro 2.

En la figura 4 se observa la ubicación de los cambios encontrados en el presente estudio en el gen USH2A.

\section{Discusión}

A partir de una tamización nacional realizada en once ciudades principales del país, para la

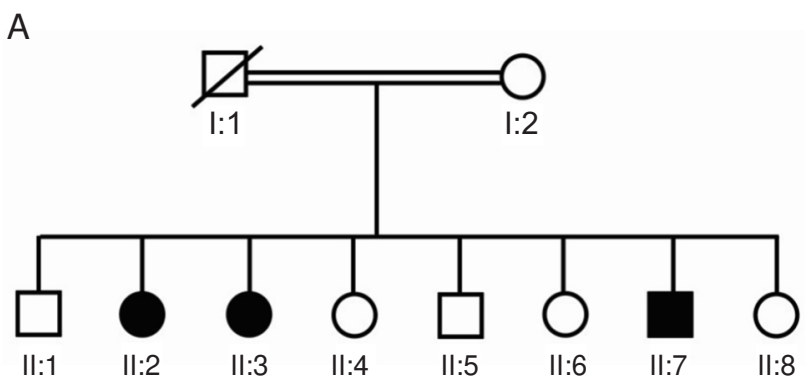




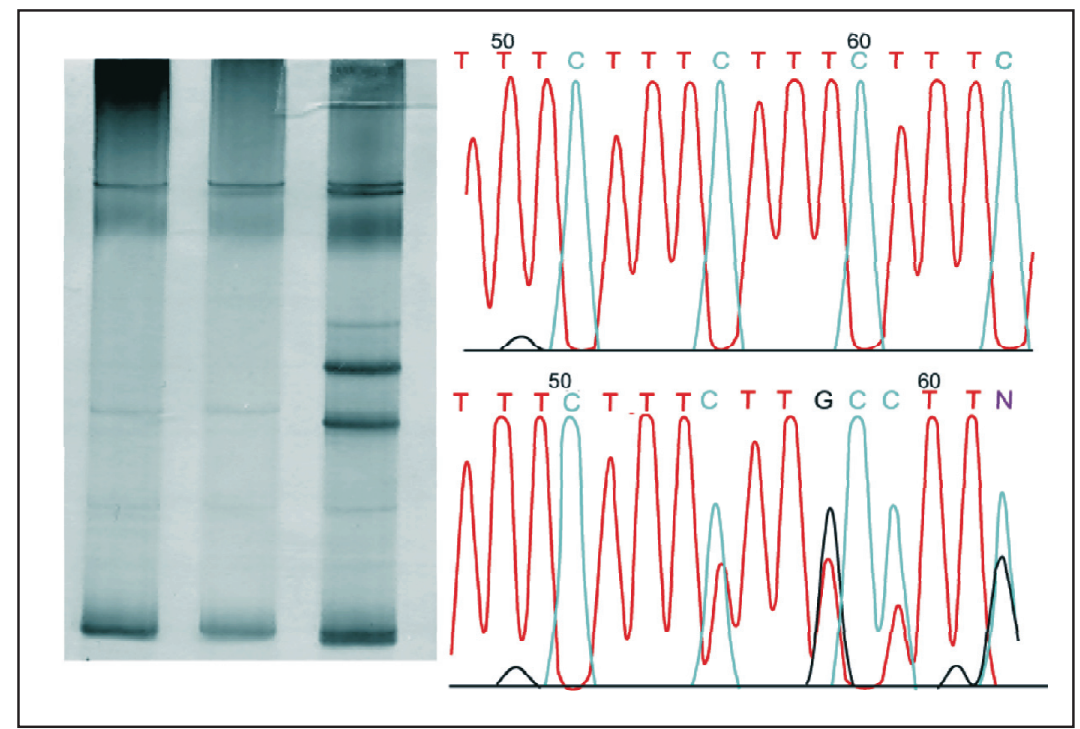

Figura 3. Cambio encontrado en el exón 20. A la izquierda el análisis de SSCP con el patrón anormal en el tercer carril. A la derecha, el electroferograma de la secuencia de tipo silvestre, en la parte superior, y de la deleción en estado heterocigoto, en la parte inferior.

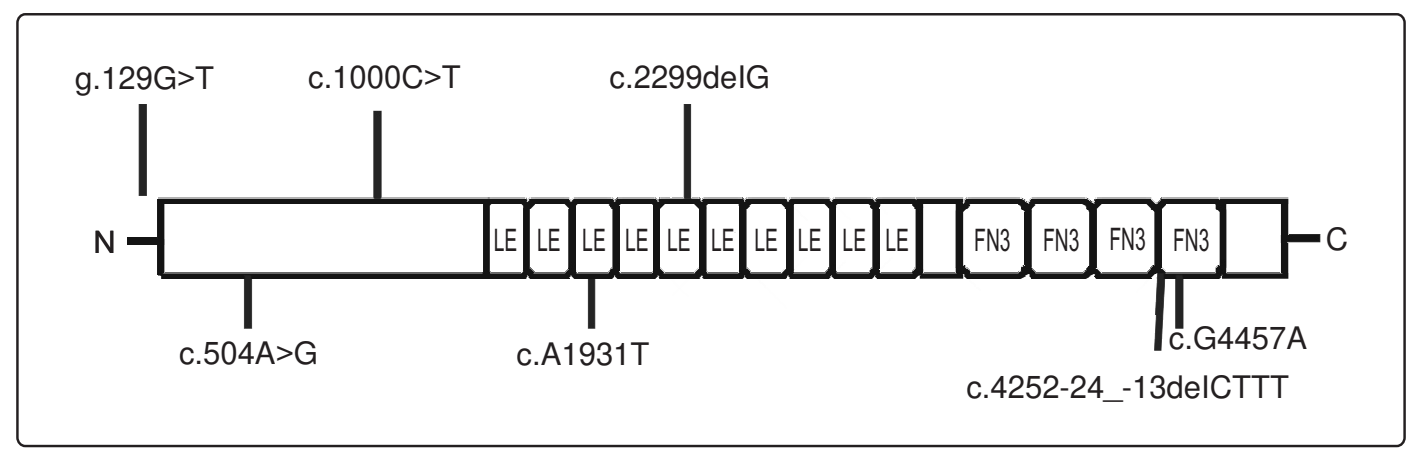

Figura 4. Representación esquemática del gen USH2A. Se observa la localización de los cambios encontrados en este estudio. En la parte superior se encuentran las mutaciones y en la parte inferior los polimorfismos.

23). Esta mutación se ha detectado en $25 \%$ de la población española con síndrome de Usher, tipo 2 (24). En el presente estudio, se detectó en $27 \%$ (7 126) de la población analizada, un porcentaje muy similar al reportado en población española.

De los siete individuos en quienes se identificó esta deleción, sólo uno la presentaba en estado homocigoto, dos la presentaban en estado heterocigoto compuesto con la mutación p.R334W y en cuatro casos se identificó en estado heterocigoto, quedando pendiente por definir la mutación en el otro alelo. El fenotipo fue muy similar y homogéneo de síndrome de Usher típico en todos los individuos con la mutación c.2299delG, a excepción de uno de ellos, quien presentaba un hallazgo poco usual en casos con el de tipo 2: una hipoacusia profunda bilateral. Aunque esta profundidad es clásica del síndrome de Usher, tipo 1, este individuo se clasificó como de tipo II debido a que no presentaba alteración vestibular y la retinitis pigmentaria había aparecido a finales de la segunda década de la vida.

En este caso, es posible que la otra mutación que aún no ha sido identificada en el otro alelo, haya producido un fenotipo auditivo más grave que en los demás afectados. También, cabría hipotetizar que pudo haber existido otro hecho externo, no genético, que hubiera profundizado su sordera (por ejemplo, otitis a repetición), pero, es difícil de comprobar dada la poca información disponible en la historia clínica.

La mutación p.R334W, identificada en el exón 6, ya había sido reportada previamente (17). En la publicación de la referencia se identificó en estado 
homocigoto en seis miembros afectados de una familia de Marruecos, en quienes se observó variabilidad fenotípica (hipoacusia progresiva y edad variable de aparición de la retinitis pigmentaria). En la población colombiana, la mutación p.R334W se identificó en estado homocigoto en dos individuos quienes presentaron, uno, hipoacusia moderada $y$, el otro, de moderada a severa (sic.), pero en ninguno de los dos progresiva. En ambos, la retinitis pigmentaria se inició en la segunda década de la vida y, en general, con hallazgos muy similares y clásicos de retinitis pigmentaria del síndrome de Usher, tipo II. Esto podría sugerir que la misma mutación esté causando fenotipos diferentes, aunque también puede estar siendo influenciada por el componente genético de cada individuo.

Si se observa la frecuencia de los alelos en la población estudiada, la mutación p.R334W (6/52 alelos) es casi tan frecuente ( 11\%) como la c. 2299 delG (8/52 alelos) ( $15 \%)$, aunque en otras poblaciones su frecuencia no es tan significativa: dos alelos en una cohorte de Estados Unidos y Gran Bretaña (11), ningún alelo en una cohorte de 36 individuos en el Reino Unido (23) y un alelo en una cohorte de 31 individuos noruegos y daneses (22). En vista de la similitud que existe en las frecuencias de estas dos mutaciones en la población colombiana, la mutación p.R334W es la segunda mutación más frecuente para el fenotipo 2 en esta población.

Con respecto al cambio g.129G $>$ T, identificado en la región promotora del gen, aún no se ha descrito en la literatura. Es bien conocido que el promotor de cualquier gen juega un papel muy importante en la regulación de su expresión. Se ha demostrado que las mutaciones en la región reguladora interrumpen el proceso normal de activación génica e iniciación de la transcripción, y pueden actuar ya sea incrementando o disminuyendo el nivel de ARNm. Las mutaciones descritas en regiones reguladoras se encuentran en las secuencias flanqueantes 5' que contienen elementos promotores constitutivos, enhancers, represores, determinantes de expresión génica específica para tejido y otros elementos reguladores (25). La detección de mutaciones en elementos reguladores potenciales desconocidos, puede predecir la existencia de tales elementos; de ahí la importancia del cambio detectado en este estudio.

Según la base de datos Genomatix (www.genomatix. de), el sitio específico donde se encuentra el cambio no parece ser relevante, por lo que éste no afectaría la expresión de la proteína. Sin embargo, este cambio se encuentra en la región flanqueante 5' del gen, se está segregando con la enfermedad en esta familia y no fue detectado en 100 controles sanos colombianos.

Todas estas razones apoyan la hipótesis de que se trata de una mutación y no de un polimorfismo. Hasta el momento, no se han reportado mutaciones de este tipo en el gen USH2A, por lo que el cambio identificado en este estudio podría ser la primera mutación reportada y representa una frecuencia de $4 \%$ en la población analizada. Algunos estudios que hacen identificación de las mutaciones del gen, excluyen la región promotora y sólo analizan la región codificante $(10,17,26)$, por lo que es posible que los cambios en esta región no estén siendo detectados en otras poblaciones.

Se necesitan posteriores estudios de expresión para establecer la naturaleza del cambio. Aunque todas estas razones apoyan la idea de que el cambio g.129G > T pueda ser una mutación, también se puede atribuir la segregación coexistente entre este cambio y la enfermedad a un posible desequilibrio de ligamiento con otra mutación no detectada y que podría existir en la porción no estudiada del gen USH2A, lo cual tendría que descartarse analizando la isoforma larga.

Se identificaron cuatro cambios polimórficos, tres de ellos previamente reportados. El primero, c.504A>G, p.Thr168Thr en el exón 3, que corresponde al polimorfismo de un único nucleótido (Single Nucleotide Polymorphism, SNP) rs4253963 (12); el segundo, el polimorfismo identificado en el exón 11, c.1931A>T, p.D644V, que corresponde al SNP rs1805048 (12); y un tercer polimorfismo adicional en el exón 21, c.4457G>A, p.R1486K, que corresponde al SNP rs1805049 (12).

El cambio identificado en el exón 20 no ha sido reportado previamente. Aunque este cambio no se identificó en 100 controles sanos, tampoco afecta un sitio de splicing o ramificación en el intrón, lo que lo definiría como un cambio que no es patógeno. La secuencia involucrada contiene seis repeticiones CTTT en tándem, de las cuales, se pierden tres con la deleción. Por sus características parece que se tratara de un STR, pero no ha sido reportado ni se encuentra en población normal. Este cambio no parece causar efecto alguno en la proteína, aunque se necesitan posteriores estudios para esclarecer su naturaleza.

En total, se logró establecer que $38 \%$ de la población presenta alguna mutación en la isoforma 
corta del gen de la usherina. En un estudio de población francesa, que se llevó a cabo con una metodología muy similar, se reportó la presencia de mutaciones en $39 \%$ de la población con síndrome de Usher, tipo 2 (25), una frecuencia muy cercana a la reportada en este estudio, pero con una mayor heterogeneidad de mutaciones identificadas. Estudios similares, pero realizados mediante secuenciación, han revelado la presencia de alguna mutación patológica en $87 \%$ de la población en países del norte de Europa (22) y en $57 \%$ de la de Estados Unidos (27), frecuencias más altas que la reportada en este estudio (38 $\%)$ debido tal vez a la técnica utilizada, ya que el análisis de secuenciación resulta ser más acertado aunque más costoso que el análisis de SSCP (28). Además, uno de los estudios reporta una frecuencia alélica de $39 \%$ de la mutación 2299delG (22), una frecuencia mayor que la reportada en éste y en otros estudios en población europea. Se ha reportado que la distribución de esta mutación varía geográficamente en Europa, disminuyendo de norte a sur (29), de ahí que se identifique en mayor frecuencia en países del norte, como Escandinavia, que en países del sur, como Francia y España. En población japonesa su frecuencia es baja (30).

Un análisis posterior de la isoforma larga del gen permitirá conocer nuevas mutaciones en estos individuos y genotipificar a una mayor proporción de la población, ya que se ha reportado que esta isoforma puede albergar incluso más mutaciones que la isoforma corta $(26,27,30,31)$. Se ha propuesto la técnica de microarrays como un método de genotipificación rápido y efectivo (32), pero, para poder llevarlo a cabo, es necesario tener una mejor idea de las mutaciones propias de población colombiana.

En conclusión, este estudio demuestra que $27 \%$ de la población analizada con síndrome de Usher, tipo 2, (26 individuos) porta la mutación c.2299delG, y esta frecuencia es muy similar a la reportada en España, que es de $25 \%$ (24).

La mutación p.R334W es la segunda mutación más frecuente en la población analizada (15\%), a diferencia de las frecuencias reportadas en otras poblaciones. El cambio g.129G $>$ T, identificado en la región promotora del gen, no ha sido previamente reportado y fue clasificado como mutación por sus características, pero representa una baja frecuencia en la población analizada (4\%). Se identificaron tres polimorfismos previamente reportados y uno adicional que no ha sido reportado, pero fue clasificado como tal por sus características.

Los resultados de este estudio revelan interesantes hallazgos y evidencian algunas diferencias con respecto a frecuencias de mutaciones reportadas en otras poblaciones. Estos resultados demuestran la importancia de establecer un panel de mutaciones propio de la población colombiana, para hacer un diagnóstico más acertado. Además, se resalta la importancia de adelantar estudios de expresión para definir la naturaleza del cambio g.129G>T.

\section{Agradecimientos}

Agradecemos al Hospital Universitario San Ignacio de Bogotá y a Vicente Rodríguez Montoya, por las valoraciones audiológicas. A la Fundación Oftalmológica Nacional, a David Medina y a Silvia Flórez, por las evaluaciones oftalmológicas, y a Martha Moncayo por los electrorretinogramas. A William Kimberling y Richard Smith, de la Universidad de lowa, Estados Unidos. Especial agradecimiento a los pacientes y a sus familias por participar en nuestro programa nacional de investigación del síndrome de Usher.

\section{Conflicto de intereses}

No se tiene ningún conflicto de intereses con autoridades públicas o privadas para su publicación.

\section{Financiación}

Este trabajo hace parte del proyecto "Estudios clínicos y moleculares en familias colombianas con síndrome de Usher, fase IV", financiado por Colciencias (código 1203-04-11732) y el Instituto de Genética Humana, Pontificia Universidad Javeriana de Bogotá (código 1045).

\section{Referencias}

1. Boughman JA, Vernon M, Shaver KA. Usher syndrome: Definition and estimate of prevalence from two high-risk populations. J Chronic Dis. 1983;36:595-603.

2. Keats BJ, Corey DP. The usher syndromes. Am J Med Genet. 1999;89:158-66.

3. Hallgren B. Retinitis pigmentosa combined with congenital deafness; with vestibulo-cerebellar ataxia and mental abnormality in a proportion of cases: A clinical and geneticostatistical study. Acta Psychiatr Scand 1959;34(Suppl.):1101.

4. Tamayo M, Bernal JE, Tamayo GE, Frias JL. Study of the etiology of deafness in an institutionalized population in Colombia. Am J Med Genet. 1992;44:405-8.

5. Tamayo ML, Bernal JE, Tamayo GE, Frias JL, Alvira G, Vergara 0 , et al. Usher syndrome: Results of a screening program in Colombia. Clin Genet. 1991;40:304-11. 
6. Tamayo ML, Maldonado C, Plaza SL, Alvira GM, Tamayo GE, Zambrano M, et al. Neuroradiology and clinical aspects of Usher syndrome. Clin Genet. 1996;50:126-32.

7. Pakarinen L, Tuppurainen K, Laippala $\mathbf{P}$, Mantyjarvi M, Puhakka H. The ophthalmological course of Usher syndrome type III. Int Ophthalmol. 1995;19:307-11.

8. Eudy JD, Weston MD, Yao S, Hoover DM, Rehm HL, MaEdmonds M, et al. Mutation of a gene encoding a protein with extracellular matrix motifs in Usher syndrome type Ila. Science. 1998;280:1753-7.

9. Liu X, Bulgakov OV, Darrow KN, Pawlyk B, Adamian M, Liberman MC, et al. Usherin is required for maintenance of retinal photoreceptors and normal development of cochlear hair cells. Proc Natl Acad Sci USA. 2007;104:4413-8.

10. Hope Cl, Bundey S, Proops D, Fielder AR. Usher syndrome in the city of Birmingham--prevalence and clinical classification. Br J Ophthalmol. 1997;81:46-53.

11. Ouyang XM, Hejtmancik JF, Jacobson SG, Li AR, Du LL, Angeli $\mathrm{S}$, et al. Mutational spectrum in Usher syndrome type II. Clin Genet. 2004;65:288-93.

12. Rosenberg T, Haim M, Hauch AM, Parving A. The prevalence of Usher syndrome and other retinal dystrophyhearing impairment associations. Clin Genet. 1997; 51: 314-21.

13. Pieke-Dahl S, Ohlemiller KK, McGee J, Walsh EJ, Kimberling WJ. Hearing loss in the RBF/DnJ mouse, a proposed animal model of Usher syndrome type Ila. Hear Res. 1997;112:1-12.

14. Weston MD, Eudy JD, Fujita S, Yao S, Usami S, Cremers C, et al. Genomic structure and identification of novel mutations in usherin, the gene responsible for Usher syndrome type Ila. Am J Hum Genet. 2000;66:1199-210.

15. Aller E, Jaijo T, Beneyto M, Najera C, Oltra S, Ayuso C, et al. Identification of 14 novel mutations in the long isoform of USH2A in Spanish patients with Usher syndrome type II. J Med Genet. 2006;43:e55.

16. Baux D, Larrieu L, Blanchet C, Hamel C, Ben Salah S, Vielle A, et al. Molecular and in silico analyses of the fulllength isoform of usherin identify new pathogenic alleles in Usher type II patients. Hum Mutat. 2007;28:781-9.

17. van Wijk E, Pennings RJ, te Brinke $H$, Claassen $A$, Yntema HG, Hoefsloot LH, et al. Identification of 51 novel exons of the Usher syndrome type 2A (USH2A) gene that encode multiple conserved functional domains and that are mutated in patients with Usher syndrome type II. Am J Hum Genet. 2004;74:738-44.

18. Adato A, Weston MD, Berry A, Kimberling WJ, BonneTamir A. Three novel mutations and twelve polymorphisms identified in the USH2A gene in Israeli USH2 families. Hum Mutat. 2000;15:388.

19. Nelson MR, Marnellos G, Kammerer S, Hoyal CR, Shi MM, Cantor CR, et al. Large-scale validation of single nucleotide polymorphisms in gene regions. Genome Res. 2004;14:1664-8.
20. Spandau UH, Rohrschneider K. Prevalence and geographical distribution of Usher syndrome in Germany. Graefes Arch Clin Exp Ophthalmol. 2002;240:495-8.

21. Bernal S, Ayuso C, Antinolo G, Gimenez A, Borrego S, Trujillo MJ, et al. Mutations in USH2A in Spanish patients with autosomal recessive retinitis pigmentosa: high prevalence and phenotypic variation. J Med Genet. 2003;40:e8.

22. Dreyer B, Tranebjaerg L, Rosenberg T, Weston MD, Kimberling WJ, Nilssen $\mathbf{O}$. Identification of novel USH2A mutations: Implications for the structure of USH2A protein. Eur J Hum Genet. 2000;8:500-6.

23. Leroy BP, Aragón-Martín JA, Weston MD, Bessant DA, Willis C, Webster AR, et al. Spectrum of mutations in USH2A in British patients with Usher syndrome type II. Exp Eye Res. 2001;72:503-9.

24. Beneyto MM, Cuevas JM, Millan JM, Espinos C, Mateu E, González-Cabo P, et al. Prevalence of 2314delG mutation in Spanish patients with Usher syndrome type II (USH2). Ophthalmic Genet. 2000;21:123-8.

25. Maubaret C, Griffoin JM, Arnaud B, Hamel C. Novel mutations in MYO7A and USH2A in Usher syndrome. Ophthalmic Genet. 2005;26:25-9.

26. Dai H, Zhang X, Zhao X, Deng T, Dong B, Wang J, et al. Identification of five novel mutations in the long isoform of the USH2A gene in Chinese families with Usher syndrome type II. Mol Vis. 2008;14:2067-75.

27. McGee TL, Seyedahmadi BJ, Sweeney MO, Dryja TP, Berson EL. Novel mutations in the long isoform of the USH2A gene in patients with Usher syndrome type II or nonsyndromic retinitis pigmentosa. J Med Genet. 2010;47:499506.

28. Rohlin A, Wernersson J, Engwall Y, Wiklund L, Bjork $\mathbf{J}$, Nordling M. Parallel sequencing used in detection of mosaic mutations: Comparison with four diagnostic DNA screening techniques. Hum Mutat. 2009;30:1012-20.

29. Aller E, Larrieu L, Jaijo T, Baux D, Espinos C, GonzálezCandelas F, et al. The USH2A c.2299delG mutation: Dating its common origin in a Southern European population. Eur $\mathrm{J}$ Hum Genet. 2010;18:788-93.

30. Nakanishi H, Ohtsubo M, Iwasaki S, Hotta Y, Mizuta $\mathrm{K}$, Mineta $\mathbf{H}$, et al. Identification of 11 novel mutations in USH2A among Japanese patients with Usher syndrome type 2. Clin Genet. 2009;76:383-91.

31. Dreyer B, Brox V, Tranebjaerg L, Rosenberg T, Sadeghi AM, Moller C, et al. Spectrum of USH2A mutations in Scandinavian patients with Usher syndrome type II. Hum Mutat. 2008;29:451.

32. Jaijo T, Aller E, García-García G, Aparisi MJ, Bernal $\mathrm{S}$, Ávila-Fernández $\mathrm{A}$, et al. Microarray-based mutation analysis of 183 Spanish families with Usher syndrome. Invest Ophthalmol Vis Sci. 2010;51:1311-7. 